\title{
Fibration categories are fibrant relative categories
}

\author{
LENNART MEIER
}

\begin{abstract}
A relative category is a category with a chosen class of weak equivalences. Barwick and Kan produced a model structure on the category of all relative categories, which is Quillen equivalent to the Joyal model structure on simplicial sets and the Rezk model structure on simplicial spaces. We will prove that the underlying relative category of a model category or even a fibration category is fibrant in the Barwick-Kan model structure.
\end{abstract}

18D99, 55U10, 55U35

\section{Introduction}

Abstract homotopy theory comes nowadays in a variety of flavors. A traditional and very rich version is Quillen's theory of model categories, first developed in [16]. More recently, various versions of $\infty$-categories, like Joyal's quasicategories and Rezk's complete Segal spaces, came into fashion. We will concentrate in this article on maybe the most naive flavor: relative categories.

A relative category is a category with a chosen class of morphisms, called weak equivalences, closed under composition and containing all identities. Despite the apparent simplicity of the definition, only recently Barwick and Kan developed in [3] a satisfactory homotopy theory of relative categories by exhibiting a model structure on the category RelCat of (small) relative categories. This model category is Quillen equivalent to the Joyal model structure on simplicial sets and the Rezk model structure on simplicial spaces.

More precisely, Barwick and Kan consider functors

$$
N, N_{\xi}: \text { RelCat } \rightarrow \text { ssSet }
$$

into simplicial spaces, where $N$ is the Rezk classifying diagram and $N_{\xi}$ is a variant of it, involving double-subdivision. They lift the Rezk model structure from ssSet to RelCat along $N_{\xi}$. This is a more complicated analog of the Thomason model structure on the category Cat of (small) categories, which is lifted from the standard model structure on sSet along $\mathrm{Ex}^{2}$ Nerve. 
Both in the Joyal and in the Rezk model structure the fibrant objects deserve special attention: These are called quasicategories and complete Segal spaces, respectively. An equally good understanding of the fibrant objects in the Barwick-Kan model structure on RelCat remains elusive to this day. We will prove, however, a sufficient criterion for fibrancy.

Main Theorem The underlying relative category of a fibration category $\mathcal{M}$ is fibrant in the Barwick-Kan model structure.

Recall to that purpose that a fibration category is a generalization of a model category, having just fibrations and weak equivalences and no cofibrations. We will use the term essentially in the sense of catégories dérivables à gauche in Cisinski [7]. Actually, we will prove that every homotopically full subcategory of a fibration category is fibrant as a relative category. Here, a homotopically full subcategory is a full subcategory closed under the relation of weak equivalence.

In [4], Barwick and Kan show via a Yoneda-type embedding that every relative category is weakly equivalent to a homotopically full subcategory of a model category. Therefore, our results imply that they actually construct an explicit fibrant replacement functor in RelCat. Our main result also allows a rather simple construction of the quasicategory associated to a model category (see Remark 2.8).

Our main result is equivalent to the statement that $N_{\xi} \mathcal{M}$ is a complete Segal space for $\mathcal{M}$ a fibration category. Rezk [18], Bergner [5] and Barwick and Kan [4] showed that a Reedy fibrant replacement of $N \mathcal{M}$ is a complete Segal space if $\mathcal{M}$ is a (partial) model category. This was generalized in Low and Mazel-Gee [13] and using this, Low showed in [12] that the analogous statement is also true for a fibration category $\mathcal{M}$. As Barwick and Kan showed in [3] that there is a Reedy equivalence $N \mathcal{M} \rightarrow N_{\xi} \mathcal{M}$ for any relative category $\mathcal{M}$, it remained to show that $N_{\xi} \mathcal{M}$ is Reedy fibrant. This is the contribution of the present paper.

Theorem If $\mathcal{M}$ is a fibration category, then $N_{\xi} \mathcal{M}$ is Reedy fibrant.

Our proof uses ideas from Meier and Ozornova [15], where we show that the category of weak equivalences of a partial model category is fibrant in the Thomason model structure. In the case of a fibration category we will actually give a simpler proof of this result in Section 4.

Note that Barwick and Kan [3] also describes a conjugate version of the Barwick-Kan model structure, using a conjugate definition of double-subdivision. The underlying 
relative categories of cofibration categories are fibrant objects in this conjugate model structure.

We give a short overview of the structure of the article. In Section 2, we will recall notation and concepts from the theory of relative categories. In Section 3, we will treat fibration categories and homotopy limits in them. In Section 4, we will give the main steps of our proofs of the two theorems above. In Section 5, we will provide a proof for the fibrancy criterion used in Section 4. In Section 6, we will give some leftover proofs about the contractibility of certain subsets of simplices.

Acknowledgements This note grew out of collaboration with Viktoriya Ozornova. I thank her for many helpful discussions, for reading earlier versions of this material and for the resulting suggestions that substantially improved the exposition and content of this paper. I also thank Zhen Lin Low for a helpful email exchange and the referee for thoughtful remarks.

\section{Homotopy theory of (relative) categories}

In this section, we will recall the definition of the Thomason model structure on the category of small categories and of the Barwick-Kan model structure on the category of small relative categories.

Thomason constructed the following model structure on the category of small categories Cat.

Theorem 2.1 [22] There is a model structure on Cat, where a map $f$ is a weak equivalence/fibration if and only if $\operatorname{Ex}^{2} \operatorname{Nerve}(f)$ is a weak equivalence/fibration. Equivalently, $f$ is a weak equivalence if and only if $\operatorname{Nerve}(f)$ is a weak equivalence.

Here, Ex denotes the right adjoint of the subdivision functor Sd: sSet $\rightarrow$ sSet. The functor Nerve: Cat $\rightarrow$ sSet has a left adjoint $c:$ sSet $\rightarrow$ Cat, called the fundamental category functor. For example, $c \Delta[n]=\underline{n}$, the category of $n$ composable morphisms. This defines a Quillen equivalence

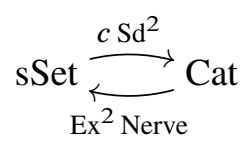

to the Kan model structure on simplicial sets.

Barwick and Kan construct an analogous model structure on the category of small relative categories. 
Definition 2.2 A relative category $\mathcal{M}$ is a category $\mathcal{M}$ together with a subcategory we $\mathcal{M}$ containing all objects of $\mathcal{M}$. The morphisms in we $\mathcal{M}$ are usually called weak equivalences. A relative functor between relative categories $\mathcal{M}$ and $\mathcal{M}^{\prime}$ is a functor $F: \mathcal{M} \rightarrow \mathcal{M}^{\prime}$ with $F($ we $\mathcal{M}) \subset$ we $\mathcal{M}^{\prime}$. We denote the category of (small) relative categories with relative functors between them by RelCat.

Remark 2.3 As we want later to view model categories as objects in RelCat, the usual size issues come up. Two possible solutions are sketched in the introduction of [15] and a more extensive treatment can be found in [20]. We will ignore these issues in the rest of this article.

Given a relative category $(\mathcal{M}$, we $\mathcal{M})$, we denote by $\operatorname{Ho}(\mathcal{M})$ its homotopy category, ie the localization of $\mathcal{M}$ at we $\mathcal{M}$. Given a category $\mathcal{C}$, we denote by $\hat{\mathcal{C}}$ its maximal relative structure, where every morphism is a weak equivalence, and by $\check{\mathcal{C}}$ its minimal relative structure, where only identities are weak equivalences.

We want to define functors $N$ and $N_{\xi}$ from RelCat to the category of simplicial spaces ssSet, where we mean by a simplicial space a bisimplicial set. To this purpose, we first have to talk about subdivision of relative posets, ie posets with the structure of a relative category. In the following, let $\mathcal{P}$ be a relative poset.

Definition 2.4 (terminal and initial subdivisions) The terminal (resp. initial) subdivision of $\mathcal{P}$ is the relative poset $\xi_{t} \mathcal{P}$ (resp. $\xi_{i} \mathcal{P}$ ) which has

(1) as objects the relative functors $\underline{n} \rightarrow \mathcal{P}$ that are monomorphisms, for $n \geq 0$,

(2) as maps $\left(x_{1}: \underline{\underline{n}}_{1} \rightarrow \mathcal{P}\right) \rightarrow\left(x_{2}: \underline{\underline{n}}_{2} \rightarrow \mathcal{P}\right)$ the commutative diagrams of the form

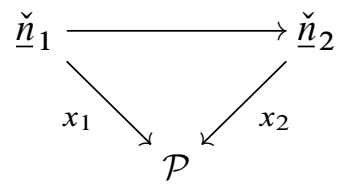

for the terminal subdivision and the commutative diagrams of the form

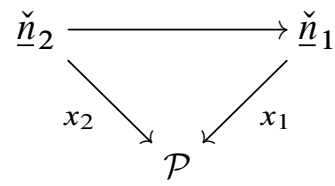

for the initial subdivision,

(3) as weak equivalences those of the above diagrams for which the induced map $x_{1}\left(n_{1}\right) \rightarrow x_{2}\left(n_{2}\right)$ (resp. $\left.x_{2}(0) \rightarrow x_{1}(0)\right)$ is a weak equivalence in $\mathcal{P}$.

The double-subdivision $\xi \mathcal{P}$ is defined as $\xi_{t} \xi_{i} \mathcal{P}$. 
In other words: The subdivision has as objects ascending chains in $\mathcal{P}$ and the terminal and initial versions correspond to two ways these can be partially ordered. For the terminal subdivision, the last-vertex map

$$
\xi_{t} \mathcal{P} \rightarrow \mathcal{P}, \quad(x: \underline{\check{n}} \rightarrow \mathcal{P}) \mapsto x(n)
$$

detects the weak equivalences. For the initial subdivision, the initial-vertex map

$$
\xi_{i} \mathcal{P} \rightarrow \mathcal{P}, \quad(x: \underline{\check{n}} \rightarrow \mathcal{P}) \mapsto x(0)
$$

detects the weak equivalences. Composing the last- and initial-vertex maps defines a natural transformation $\xi \rightarrow$ id.

Example 2.5 Let $\underline{n}$ be equipped with an arbitrary relative structure. An object of $\xi(\underline{n})$ can be identified with an ascending nonempty chain of nonempty subsets of $\{0, \ldots, n\}$. If we can build a chain

$$
A_{\bullet}=\left(A_{0} \subsetneq \cdots \subsetneq A_{m}\right)
$$

from a chain

$$
B_{\bullet}=\left(B_{0} \subsetneq \cdots \subsetneq B_{l}\right)
$$

by adding subsets, then $B_{\bullet} \leq A_{\bullet}$. The corresponding morphism is a weak equivalence if $\phi\left(A_{\bullet}\right) \simeq \phi\left(B_{\bullet}\right)$ in $\underline{n}$, where $\phi$ is the functor $\xi(\underline{n}) \rightarrow \underline{n}$ sending a chain $A$. to the smallest element of $A_{0}$.

Note here that $A_{0}$ is the largest element in the chain $A_{\bullet}$ in $\xi_{i} \underline{n}$, but our numbering system seems more natural to the author than the opposite one. Note furthermore that Nerve $\xi(\underline{n})$ is isomorphic to the double barycentric subdivision $\operatorname{Sd}^{2} \Delta[n]$. It follows that the underlying category of $\xi(\underline{n})$ is isomorphic to $c \mathrm{Sd}^{2} \Delta[n]$ as $c$ Nerve $\cong \mathrm{id}_{\text {Cat }}$.

Next, we define the classifying diagram of a relative category, an analog of the nerve functor.

Definition 2.6 For a relative category $\mathcal{M}$, we define its classifying diagram to be the simplicial space $N \mathcal{M}$ with $(N \mathcal{M})_{p q}=\operatorname{RelCat}(\underline{\underline{p}} \times \underline{\hat{q}}, \mathcal{M})$. Likewise, we define $N_{\xi} \mathcal{M}$ to be the simplicial space with $\left(N_{\xi} \mathcal{M}\right)_{p q}=\operatorname{Rel} \operatorname{Cat}(\xi(\underline{\underline{p}} \times \underline{\hat{q}}), \mathcal{M})$.

The natural transformation $\xi \rightarrow$ id induces a natural weak equivalence $N \rightarrow N_{\xi}$, as shown in [3, Lemma 5.4].

Rezk defines in [18] a model structure on the category of simplicial spaces ssSet, where the fibrant objects are the complete Segal spaces. He constructs it as a localization of the usual Reedy model structure. Barwick and Kan lift their model structure on RelCat from the Rezk model structure on ssSet. 
Theorem 2.7 [3] There is a model structure on RelCat, where a map $f$ is a weak equivalence/fibration if and only if $N_{\xi} f$ is a weak equivalence/fibration in the Rezk model structure on ssSet.

Note here that $N_{\xi} f$ is a weak equivalence if and only if $N f$ is one, but there is no analogous statement for fibrations. Barwick and Kan also show in [2] that $f: \mathcal{M} \rightarrow \mathcal{N}$ is a weak equivalence in RelCat if and only if it induces a Dwyer-Kan equivalence of the hammock localizations

$$
L^{H} \mathcal{M} \rightarrow L^{H} \mathcal{N}
$$

ie an equivalence of homotopy categories and weak equivalences of mapping spaces.

The functors

$$
N, N_{\xi}: \text { RelCat } \rightarrow \text { ssSet }
$$

have left adjoints $K$ and $K_{\xi}$, respectively. These are the unique colimit-preserving functors with $K(\Delta[p, q])=\underline{\underline{p}} \times \underline{\hat{q}}$ and $K_{\xi}(\Delta[p, q])=\xi(\check{\underline{p}} \times \underline{\hat{q}})$, respectively. Here, $\Delta[p, q]$ is the bisimplicial set whose $m$-n-simplices $\Delta[p, \bar{q}]_{m n}$ form the set of maps $(m, n) \rightarrow(p, q)$ in $\Delta \times \Delta$. We have diagrams

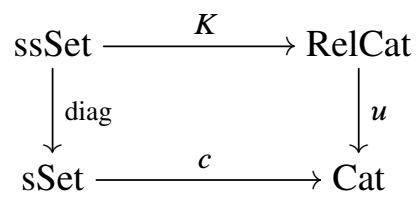

and

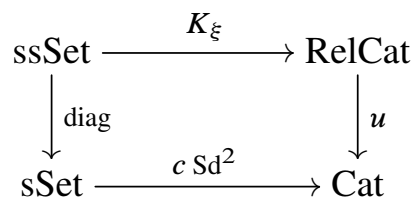

that are commutative up to natural isomorphism, where $u$ denotes the forgetful functor and $\operatorname{diag}\left(X_{\bullet .}\right)_{m}=X_{m m}$. Indeed, we have

$$
\begin{aligned}
u K \Delta[p, q] \cong \underline{p} \times \underline{q} \cong c \operatorname{Nerve}(\underline{p} \times \underline{q}) \cong c(\Delta[p] \times \Delta[q]) \cong c \operatorname{diag} \Delta[p, q], \\
u K_{\xi} \Delta[p, q] \cong u \xi(\underline{p} \times \underline{\hat{q}}) \cong c \operatorname{Sd}^{2}(\Delta[p] \times \Delta[q]) \cong c \operatorname{Sd}^{2} \operatorname{diag} \Delta[p, q],
\end{aligned}
$$

and $u$ and diag are both colimit-preserving as $u$ is left adjoint to the functor $\mathcal{C} \mapsto \hat{\mathcal{C}}$.

Remark 2.8 The functor $N_{\xi}$ : RelCat $\rightarrow$ ssSet is actually a right Quillen equivalence from the Barwick-Kan model structure to the Rezk model structure, as shown in [3, 
Theorem 6.1]. There is a further Quillen equivalence

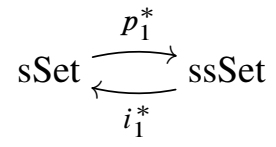

between the Joyal model structure and the Rezk model structure, as shown in [11]. The functor $i_{1}^{*}$ assigns to a bisimplicial set $X_{\bullet \bullet}$ its zeroth row $X_{\bullet 0}$. In particular, we have

$$
\left(i_{1}^{*} N_{\xi} \mathcal{C}\right)_{p}=\operatorname{RelCat}(\xi(\underline{\check{p}}), \mathcal{C}) .
$$

If $\mathcal{C}$ is fibrant, $i_{1}^{*} N_{\xi} \mathcal{C}$ is fibrant in the Joyal model structure, ie a quasicategory (also known in [14] as an $\infty$-category). As our main theorem states that the underlying relative category of every fibration category is fibrant, this gives a model for the quasicategory associated with a fibration category.

As explained in [19], results by Toën and Barwick and Kan imply that this is equivalent to other quasicategories associated with $\mathcal{M}$, in particular to the quasicategory $N_{c}\left(L^{H} \mathcal{M}\right)^{f}$, where $L^{H}$ is the hammock localization, $f$ denotes a fibrant replacement in the Bergner model structure on simplicial categories and $N_{c}$ : sCat $\rightarrow$ sSet denotes the coherent nerve. For another convenient model for the quasicategory associated with a (co)fibration category, see [21].

\section{Fibration categories and homotopy limits}

Relative categories without extra structure are often hard to work with. Therefore, several mathematicians introduced more structured versions like model categories or fibration categories. We will work with the following definition of a fibration category:

Definition 3.1 A fibration category is a relative category $(\mathcal{M}$, we $\mathcal{M})$, together with a subcategory fib $\mathcal{M} \subseteq \mathcal{M}$ of fibrations, fulfilling the following axioms:

(F1) $\mathcal{M}$ has a terminal object $*$. We call an object $x \in \mathcal{M}$ fibrant if $x \rightarrow *$ is a fibration. We assume $*$ to be fibrant.

(F2) All isomorphisms are weak equivalences, and all isomorphisms with fibrant codomain are fibrations.

(F3) Let $f, g$ and $h$ be composable morphisms. If $g f$ and $h g$ are weak equivalences, then so are $f, g$ and $h$.

(F4) Let $f: A \rightarrow C$ be a morphism between fibrant objects. If $p: B \rightarrow C$ is a (trivial) fibration, then the pullback $B \times{ }_{C} A$ exists and the map $B \times{ }_{C} A \rightarrow A$ is also a (trivial) fibration. Here, a fibration is called trivial if it is a weak equivalence. 
(F5)

Any map $f: A \rightarrow C$ with $C$ fibrant factors as

$$
A \stackrel{s}{\longrightarrow} B \stackrel{p}{\longrightarrow} C
$$

with $s$ a weak equivalence, $p$ a fibration and $p s=f$.

This agrees essentially with the notion of catégories dérivables à gauche in the sense of [7] and with the notion of an (ABC) prefibration category from [17], only that we ask for a 2-out-of-6 axiom instead of 2-out-of-3. In the presence of the 2-out-of-3 axiom, the 2-out-of-6 axiom is equivalent to we $\mathcal{M}$ being closed under retracts and also equivalent to $(\mathcal{M}$, we $\mathcal{M})$ being saturated in the sense that we $\mathcal{M}$ consists exactly of those morphisms that become isomorphisms in $\operatorname{Ho}(\mathcal{M})$; see [17, Theorem 7.2.7]. Radulescu-Banu also discusses the relationship of our definition of fibration category with other notions of fibration categories. In particular, every model category is a fibration category by forgetting the cofibrations.

Next, we will define homotopy limits of diagrams in fibration categories indexed over an arbitrary finite inverse category. We follow the treatments in [7, Sections 1 and 2] ${ }^{1}$ and [17, Chapter 9].

We fix in the following a finite inverse category $\mathcal{D}$ and a fibration category

$$
(\mathcal{M} \text {, we } \mathcal{M} \text {, fib } \mathcal{M}) \text {. }
$$

Then there is a Reedy fibration category structure on the functor category $\mathcal{M}^{\mathcal{D}}$ with weak equivalences defined objectwise and Reedy fibrations as fibrations; see [7, Théorème 1.30].

Theorem 3.2 [7, Proposition 2.6] The constant diagram functor

$$
\text { const }_{\mathcal{D}}: \operatorname{Ho}(\mathcal{M}) \rightarrow \operatorname{Ho}\left(\mathcal{M}^{\mathcal{D}}\right)
$$

has a right adjoint holim $_{\mathcal{D}}$.

This adjoint is constructed as follows: Given a functor $F: \mathcal{D} \rightarrow \mathcal{M}$, take a Reedy fibrant replacement $F \rightarrow F^{\prime}$. The limit of $F^{\prime}$ exists and $\lim _{\mathcal{D}} F^{\prime}$ maps up to isomorphism to $\operatorname{holim}_{\mathcal{D}} F$ in the homotopy category.

Given functors $F: \mathcal{D} \rightarrow \mathcal{M}$ and $i: \mathcal{A} \rightarrow \mathcal{D}$ (with $\mathcal{A}$ finite inverse), we have an induced map

$$
u_{\mathcal{A}}^{\mathcal{D}}: \operatorname{holim}_{\mathcal{D}} F \rightarrow \operatorname{holim}_{\mathcal{A}} i^{*} F,
$$

\footnotetext{
${ }^{1}$ Beware that Cisinski uses finite direct categories as he considers presheaves instead of covariant functors. His term for a Reedy fibration is fibration bordée.
} 
adjoint to the map

$$
\text { const }_{\mathcal{A}} \operatorname{holim}_{\mathcal{D}} F \rightarrow i^{*} F
$$

that is given by applying $i^{*}$ to the counit const $_{\mathcal{D}} \operatorname{holim}_{\mathcal{D}} F \rightarrow F$. The map $u_{\mathcal{A}}^{\mathcal{D}}$ will also be called the canonical map. This is a functorial construction in the sense that $u_{\mathcal{A}}^{\mathcal{D}}=u_{\mathcal{A}}^{\mathcal{B}} \circ u_{\mathcal{B}}^{\mathcal{D}}$ for a diagram of finite inverse categories $\mathcal{A} \rightarrow \mathcal{B} \rightarrow \mathcal{D}$, as can be shown by standard properties of adjoints.

Next, we want to prove three properties of the homotopy limit construction in fibration categories. We will reduce these statements to already known results in the world of model categories via a Yoneda-type construction. This technique is by no means new and was, for example, already used by Toën and Vaquié [23] in the context of dg-categories and by Cisinski [7] in the context of fibration categories.

Proposition 3.3 Let $(\mathcal{M}$, we $\mathcal{M}$, fib $\mathcal{M})$ be a (small) fibration category. ${ }^{2}$ Then there exists a functor $h: \mathcal{M} \rightarrow \mathcal{P}_{w}(\mathcal{M})$ into a model category with functorial factorizations, which has the following properties:

(1) $h$ preserves and reflects weak equivalences.

(2) If all objects of $\mathcal{M}$ are fibrant, then $h$ preserves homotopy limits along arbitrary finite inverse categories.

Proof We will construct $h$ via a Yoneda-type embedding, following [8, Section 3]. Let $\mathcal{P}(\mathcal{M})$ be the category of simplicial presheaves on $\mathcal{M}$ with the projective model structure. Consider the Yoneda embedding $h: \mathcal{M} \rightarrow \mathcal{P}(\mathcal{M})$ (taking values in discrete simplicial sets) and define $\mathcal{P}_{w}(\mathcal{M})$ to be the Bousfield localization of $\mathcal{P}(\mathcal{M})$ at $h($ we $\mathcal{M})$. Note that $\mathcal{P}_{w}(\mathcal{M})=\mathcal{P}(\mathcal{M})$ as categories, but the model structures are different.

Clearly $h: \mathcal{M} \rightarrow \mathcal{P}_{w}(\mathcal{M})$ preserves weak equivalences. We want to show that it also detects weak equivalences. Cisinski observes that the $h$ (we $\mathcal{M})$-local objects in $\mathcal{P}(\mathcal{M})$ are exactly those presheaves $\mathcal{F}$ such that $\mathcal{F}(Y) \rightarrow \mathcal{F}(X)$ is a weak equivalence if $X \rightarrow Y$ is a weak equivalence in $\mathcal{M}$. For example, the discrete presheaf ho $X$ defined by

$$
\operatorname{ho}_{X}(Y)=\operatorname{Ho}(\mathcal{M})(Y, X)
$$

is $h$ (we $\mathcal{M}$ )-local for every $X \in \mathcal{M}$. Thus, ho $X$ is fibrant in $\mathcal{P}_{w}(\mathcal{M})$ for all $X \in \mathcal{M}$.

\footnotetext{
${ }^{2}$ The smallness hypothesis can be ensured for our purposes either by the use of universes or by the following observation: If $F: \mathcal{C} \rightarrow \mathcal{M}$ is a functor from a small category $\mathcal{C}$, then $F$ factors over a small fibration subcategory $\mathcal{M}^{\prime} \subset \mathcal{M}$; the homotopy limit of $F$, if $\mathcal{C}$ is finite inverse, can then be computed in $\mathcal{M}^{\prime}$.
} 
Assume now that for a morphism $f: X \rightarrow Y$ in $\mathcal{M}$ the morphism $h(f): h(X) \rightarrow h(Y)$ is a weak equivalence in $\mathcal{P}_{w}(\mathcal{M})$. Observe that $h(X)$ and $h(Y)$ are projectively cofibrant (because acyclic fibrations are surjective on zero-simplices) and thus also cofibrant in $\mathcal{P}_{w}(\mathcal{M})$. The model category $\mathcal{P}_{w}(\mathcal{M})$ is simplicial by [1, Theorem 4.46] and thus $h(f)$ induces a weak equivalence of mapping spaces

$$
\operatorname{map}\left(h(Y), \operatorname{ho}_{Z}\right) \rightarrow \operatorname{map}\left(h(X), \operatorname{ho}_{Z}\right) .
$$

As ho $Z$ is discrete, these mapping spaces are discrete as well. A weak equivalence between discrete simplicial sets is an isomorphism and thus $h(X) \rightarrow h(Y)$ induces in particular an isomorphism on the 0 -simplices of these mapping spaces, ie the corresponding Hom-sets in $\mathcal{P}(\mathcal{M})$. Using the Yoneda lemma, we get that

$$
\operatorname{Ho}(\mathcal{M})(Y, Z) \cong \operatorname{Hom}_{\mathcal{P}(\mathcal{M})}\left(h(Y), \operatorname{ho}_{Z}\right) \rightarrow \operatorname{Hom}_{\mathcal{P}(\mathcal{M})}\left(h(X), \operatorname{ho}_{Z}\right) \cong \operatorname{Ho}(\mathcal{M})(X, Z)
$$

is an isomorphism for every $Z \in \mathcal{M}$. Hence, $X \rightarrow Y$ induces an isomorphism in $\operatorname{Ho}(\mathcal{M})$ and is thus a weak equivalence by [17, Theorem 7.2.7].

Assume now that all objects of $\mathcal{M}$ are fibrant. Then by [17, Proposition 2.1.2], $\mathcal{M}$ is a category of fibrant objects in the sense of Brown. By [8, Corollaire 3.12], $h$ preserves fibrations and acyclic fibrations; furthermore, it preserves all limits. Clearly, $h$ thus preserves Reedy fibrant diagrams and hence preserves all homotopy limits along finite inverse categories.

Proposition 3.4 Let $\mathcal{M}$ be a fibration category and $\mathcal{D}$ be a finite inverse category whose nerve is contractible. Let $F: \mathcal{D} \rightarrow$ we $\mathcal{M}$ be a diagram. Then the morphism

$$
u_{d}^{\mathcal{D}}: \operatorname{holim}_{\mathcal{D}} F \rightarrow F(d)
$$

is an isomorphism in $\operatorname{Ho}(\mathcal{M})$ for every $d \in \mathcal{D}$.

Proof By a (Reedy) fibrant replacement, we can replace $F$ by a diagram in the subcategory of fibrant objects $\mathcal{M}_{\text {fib }}$ with the same homotopy limit (computed in $\mathcal{M}_{\text {fib }}$ ). Thus, we can assume that every object of $\mathcal{M}$ is fibrant. The result follows now from the corresponding result for model categories [6, Corollary 29.2, Section 31] and Proposition 3.3.

Definition 3.5 A functor $i: \mathcal{A} \rightarrow \mathcal{B}$ between two categories is called homotopically initial if $\operatorname{Nerve}(i / b)$ is (weakly) contractible for every $b \in B$, where $i / b$ denotes the comma category of pairs $(a \in \mathcal{A}, i(a) \rightarrow b)$. 
Proposition 3.6 Let $\mathcal{M}$ be a fibration category and $\mathcal{D}$ be a finite inverse category. Let $i: \mathcal{A} \rightarrow \mathcal{D}$ be a homotopically initial functor and $F: \mathcal{D} \rightarrow \mathcal{M}$ a diagram. Then the canonical map

$$
\operatorname{holim}_{\mathcal{D}} F \rightarrow \operatorname{holim}_{\mathcal{A}}(F i)
$$

is an isomorphism in $\operatorname{Ho}(\mathcal{M})$.

Proof As before, we can assume that $\mathcal{M}$ has only fibrant objects. The result follows now from the corresponding result for model categories [6, Section 31.6] and Proposition 3.3.

For the following proposition recall that a full subcategory $\mathcal{A} \subset \mathcal{D}$ is called a cosieve if for every $a \in \mathcal{A}$ and every morphism $a \rightarrow d$ in $\mathcal{D}$, we already have $d \in \mathcal{A}$.

Proposition 3.7 Let $\mathcal{M}$ be a fibration category and $\mathcal{D}$ be a finite inverse category. Let $\mathcal{A}, \mathcal{B} \subset \mathcal{D}$ be inclusions of cosieves. Let $F: \mathcal{D} \rightarrow \mathcal{M}$ be a diagram. Then there is an isomorphism

$$
\operatorname{holim}_{\mathcal{D}} F \rightarrow \operatorname{holim}_{\mathcal{A}} F \times \operatorname{holim}_{\mathcal{A} \cap \mathcal{B}}^{h} \operatorname{holim}_{\mathcal{B}} F
$$

in $\operatorname{Ho}(\mathcal{M})$, compatible with the canonical maps to $\operatorname{holim}_{\mathcal{A}} F$ and $\operatorname{holim}_{\mathcal{B}} F$.

Proof As before, we can assume that $\mathcal{M}$ has only fibrant objects. The result follows now from the corresponding result for model categories [6, Section 31.5] and Proposition 3.3 as follows: Chachólski and Scherer prove that

$$
\operatorname{holim}_{\mathcal{E}} F \rightarrow \operatorname{holim}_{\mathcal{A}} F \times \mathrm{holim}_{\mathcal{A} \cap \mathcal{B}}^{h} \operatorname{holim}_{\mathcal{B}} F
$$

is an equivalence, where $\mathcal{E}$ is a co-Grothendieck construction, which is in our case given as follows: It has objects

- $(a, 0)$ for $a \in \mathcal{A}$,

- $(b, 1)$ for $b \in \mathcal{B}$, and

- $(c, 01)$ for $c \in \mathcal{A} \cap \mathcal{B}$.

The morphisms $(d, i) \rightarrow\left(d^{\prime}, i^{\prime}\right)$ are morphisms $d \rightarrow d^{\prime}$ in $\mathcal{D}$ if $i=i^{\prime}$ or $i=0$ or 1 and $i^{\prime}=01$.

We will show that the functor

$$
G: \mathcal{E} \rightarrow \mathcal{D}, \quad(d, i) \mapsto d
$$


is homotopically initial. The category $G / d$ has

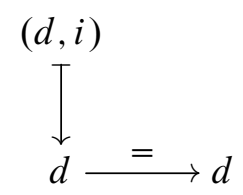

as terminal object with

- $\quad i=0$ if $d \in \mathcal{A}$, but $d \notin \mathcal{B}$,

- $\quad i=1$ if $d \in \mathcal{B}$, but $d \notin \mathcal{A}$, and

- $i=01$ if $d \in \mathcal{A} \cap \mathcal{B}$.

In the first two cases, we use that $\mathcal{A} \subset \mathcal{D}$ and $\mathcal{B} \subset \mathcal{D}$ are cosieves.

Thus,

$$
u_{\mathcal{E}}^{\mathcal{D}}: \operatorname{holim}_{\mathcal{D}} F \rightarrow \operatorname{holim}_{\mathcal{E}} F
$$

is an equivalence by Proposition 3.6 and the result follows.

Remark 3.8 Instead of using [6], we could also have used the language of quasicategories. The model category $\mathcal{P}_{w}(\mathcal{M})$ is actually simplicial by [1, Theorem 4.46]. By [14, Theorem 4.2.4.1], homotopy limits in $\mathcal{P}_{w}(\mathcal{M})$ and in the coherent nerve $N_{c} \mathcal{P}_{w}(\mathcal{M})^{\circ}$ agree. Thus, the last three propositions follow from the corresponding results in quasicategories: [14, Corollary 4.4.4.10], [14, Theorem 4.1.3.1 and Proposition 4.1.1.8] and [14, Corollary 4.2.3.10].

\section{Fibration categories are fibrant}

Our main goal in this section is to prove that $N_{\xi} \mathcal{M}$ is Reedy fibrant if $\mathcal{M}$ is a fibration category. This implies then that every fibration category is fibrant as a relative category in the Barwick-Kan model structure. In this section, we will only provide the main steps, while the following two sections contain the more technical parts.

First, we have to introduce the following notation: For a category $\mathcal{D}$, let $\mathcal{K}(\mathcal{D})$ be the category $\mathcal{D} \times(0 \rightarrow 1) \cup_{\mathcal{D} \times 1} \mathcal{D}^{\triangleleft}$, where $\mathcal{D}^{\triangleleft}$ denotes the category $\mathcal{D}$ with an additional initial object. Thus, $\mathcal{K}(\mathcal{D})$ consists of two copies of $\mathcal{D}$ with a unique map from the 0 -copy of each object to the 1-copy of it, and each object in the 1-copy receives an additional morphism from a "partial initial object". We will view $\mathcal{D}$ as a subcategory of $\mathcal{K}(\mathcal{D})$ via the identification $\mathcal{D} \cong \mathcal{D} \times 0$. We will furthermore denote the "partial initial object" by $k_{\mathcal{D}} \in \mathcal{K}(\mathcal{D})$.

In [15, Lemma 4.2], we showed the following fibrancy criterion for the Thomason model structure: 
Proposition 4.1 A category $\mathcal{C}$ is fibrant in the Thomason model structure if and only if it has the right lifting property with respect to the inclusions

$$
c \mathrm{Sd}^{2} \Lambda^{n}[n] \rightarrow \mathcal{K}\left(c \mathrm{Sd}^{2} \Lambda^{n}[n]\right) \quad \text { for } n \geq 1
$$

Our first aim is to show that the category of weak equivalences of a fibration category is fibrant in the Thomason model structure. The following proposition will be key:

Proposition 4.2 Let $\mathcal{D}$ be an arbitrary finite inverse category and $F: \mathcal{D} \rightarrow \mathcal{M}$ be a functor to a fibration category $\mathcal{M}$. Then one can extend $F$ to a functor $G: \mathcal{K}(\mathcal{D}) \rightarrow \mathcal{M}$ such that $G((d, 0) \rightarrow(d, 1))$ is a weak equivalence for every $d \in \mathcal{D}$ and $\left.G\right|_{\mathcal{D} \triangleleft}$ is a homotopy limit diagram.

Proof We can find a weak equivalence $F \rightarrow F^{\prime}$ to a Reedy fibrant diagram, corresponding to a functor $v: D \times \underline{1} \rightarrow \mathcal{M}$. As discussed in the previous section, limits of Reedy fibrant diagrams exist and are homotopy limits. Let $\widetilde{F^{\prime}}: \mathcal{D}^{\triangleleft} \rightarrow \mathcal{M}$ be a limit cone for $F^{\prime}$. Then we can glue $G$ from $v$ and $\widetilde{F^{\prime}}$.

The following corollary also follows from our later results, but we prefer to give a direct proof.

Corollary 4.3 The category of weak equivalences of a fibration category is fibrant in the Thomason model structure.

Proof The category $c \mathrm{Sd}^{2} \Lambda^{k}[n]$ is inverse. Indeed, $c \operatorname{Sd}^{2} \Lambda^{k}[n] \subset c \operatorname{Sd}^{2} \Delta[n]$ can be viewed as consisting of chains of subsets of $\underline{n}$ and the length of the chain provides the inverse structure.

Let $(\mathcal{M}$, we $\mathcal{M}$, fib $\mathcal{M})$ be a fibration category and $F: c \operatorname{Sd}^{2} \Lambda^{n}[n] \rightarrow$ we $\mathcal{M}$ be a diagram. By Proposition 4.2 and Proposition 3.4, we can extend $F$ to a diagram $\mathcal{K}\left(c \mathrm{Sd}^{2} \Lambda^{n}[n]\right) \rightarrow$ we $\mathcal{M}$. Proposition 4.1 implies the statement.

Remark 4.4 The proof in [15] of the fibrancy of partial model categories was considerably harder as no analog of Reedy fibrant replacement for functors indexed by inverse categories exists for general partial model categories.

Showing fibrancy in the Barwick-Kan model structure is more complicated than in the Thomason model structure. Before we formulate a fibrancy criterion, we have to discuss certain preliminaries. 
We can identify $\mathcal{K}\left(c \operatorname{Sd}^{2} \partial \Delta[n]\right)$ and $\xi \underline{n}$ as categories as follows: Objects in $c \operatorname{Sd}^{2} \partial \Delta[n]$ can be identified with ascending nonempty chains

$$
A_{\bullet}=\left(A_{0} \subsetneq \cdots \subsetneq A_{m}\right)
$$

of nonempty subsets of $\underline{n}$ such that $A_{m} \neq \underline{n} .{ }^{3}$ For such chains, we identify $\left(A_{\bullet}, 1\right)$ in $\mathcal{K}\left(c \mathrm{Sd}^{2} \partial \Delta[n]\right)$ with $A_{0} \subsetneq \cdots \subsetneq A_{m} \subsetneq \underline{n}$ and $k_{c \mathrm{Sd}^{2} \partial \Delta[n]}$ with the chain just consisting of $\underline{n}$ in $\xi(\underline{n})$. We refer the reader to [15, Remark 4.1] for a picture of this identification. If we choose a relative structure on $\underline{n}$, the relative structure of $\xi \underline{n}$ thus defines a relative structure on $\mathcal{K}\left(c \operatorname{Sd}^{2} \partial \Delta[n]\right)$ and thus also on $\mathcal{K}\left(c \operatorname{Sd}^{2} \Lambda^{k}[n]\right)$ for every $0 \leq k \leq n$ by restriction.

We are now ready to formulate the following fibrancy criterion that will be proved in slightly stronger form as Proposition 5.3.

Proposition 4.5 Let $\mathcal{M}$ be relative category. Assume that $\mathcal{M}$ has the right lifting property with respect to the inclusions

$$
c \mathrm{Sd}^{2} \Lambda^{k}[n] \rightarrow \mathcal{K}\left(c \mathrm{Sd}^{2} \Lambda^{k}[n]\right)
$$

for $n \geq 1$ and $0 \leq k \leq n$, where the relative structure on $\mathcal{K}\left(c \operatorname{Sd}^{2} \Lambda^{k}[n]\right)$ is induced by an arbitrary relative structure on $\underline{n}$ such that $(n-1) \rightarrow n$ is a weak equivalence if $k=n$. Then $N_{\xi} \mathcal{M}$ is Reedy fibrant.

Let now and in the following $n \geq 1$ and $0 \leq k \leq n$ be fixed numbers. Equip $\underline{n}$ with an arbitrary relative structure such that $(n-1) \rightarrow n$ is a weak equivalence if $k=n$. Set for the rest of the section $\mathcal{D}=c \operatorname{Sd}^{2} \Lambda^{k}[n]$, with relative structure induced by that on $\underline{n}$. We now want to describe the weak equivalences in $\mathcal{D}$ more concretely:

The functor

$$
\pi=\left.\phi\right|_{\mathcal{D}}: \mathcal{D} \subset \xi \underline{n} \stackrel{\phi}{\longrightarrow} \underline{n}
$$

described in Example 2.5 detects and preserves weak equivalences. This implies the following description of weak equivalences: All morphisms $\left(A_{\bullet}, 0\right) \rightarrow\left(A_{\bullet}, 1\right)$ are weak equivalences. A morphism $\left(A_{\bullet}, i\right) \rightarrow\left(B_{\bullet}, i\right)$ for $i=0$ or 1 is a weak equivalence if and only if $\pi\left(A_{\bullet}\right) \simeq \pi\left(B_{\bullet}\right)$ in $\underline{n}$. Furthermore, $k_{\mathcal{D}} \rightarrow A_{\bullet} \times 1$ is a weak equivalence if and only if $\pi\left(A_{\bullet}\right) \simeq 0$ in $\underline{n}$.

Let now and in the following $\mathcal{M}$ be a fibration category and $F: \mathcal{D} \rightarrow \mathcal{M}$ be a relative functor. To apply Proposition 4.5, we need to show that the functor $G: \mathcal{K}(\mathcal{D}) \rightarrow \mathcal{M}$ constructed in Proposition 4.2 is actually a relative functor. The following proposition implies exactly that.

\footnotetext{
${ }^{3}$ Here and in the following, we abuse notation by using $\underline{n}$ both for the category of $n$ composable morphisms and for $\{0,1, \ldots, n\}$, its set of objects.
} 
Proposition 4.6 Let $F: \mathcal{D} \rightarrow \mathcal{M}$ be a relative functor. Then $\operatorname{holim}_{\mathcal{D}} F \rightarrow F(0)$ is a weak equivalence. Here, we identify 0 with the object of $\mathcal{D}$ corresponding to the chain of subsets of $\underline{n}$ just consisting of $\{0\}$, ie with the 0 -corner.

The basic intuition is that after collapsing all weak equivalences to identities, $c \operatorname{Sd}^{2} \Lambda^{k}[n]$ becomes a quotient of $\underline{n}$ with 0 as initial object. Of course, more care has to be taken for an actual proof. We will proceed inductively over the $\pi^{-1}(\underline{i})$ for $i \leq n$ and need for that a few intermediate results. As Proposition 4.6 is clear for $n=1$, we will assume that $n \geq 2$ in the following.

Lemma 4.7 Let $i$ and $j$ be integers with $0 \leq j \leq i \leq n$. Then there is a homotopy pullback diagram

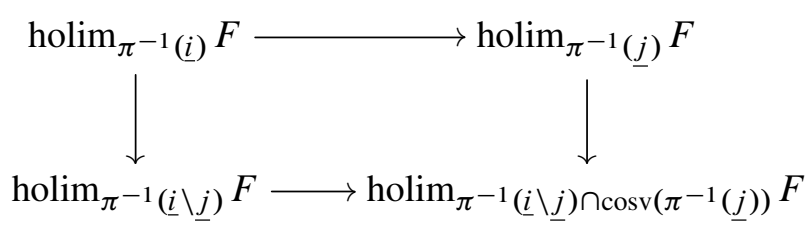

where the horizontal maps and the left vertical map are the canonical maps. Here, $\operatorname{cosv}(\mathcal{C}) \subset \mathcal{D}$ denotes for a subcategory $\mathcal{C} \subset \mathcal{D}$ the cosieve generated by $\mathcal{C}$, ie the full subcategory of all $d \in \mathcal{D}$ such that a morphism $c \rightarrow d$ in $\mathcal{D}$ with $c \in \mathcal{C}$ exists.

Proof We should first explain the right vertical map inside the homotopy pullback diagram. We claim that the canonical map

$$
\operatorname{holim}_{\pi^{-1}(i) \cap \operatorname{cosv}\left(\pi^{-1}(\underline{j})\right)} F \rightarrow \operatorname{holim}_{\pi^{-1}(\underline{j})} F
$$

is an equivalence. Indeed, we can describe the two relevant categories as

$$
\begin{array}{r}
\pi^{-1}(\underline{j})=\left\{w_{0} \subsetneq \cdots \subsetneq w_{m} \mid c \in w_{0} \text { for some } c \leq j\right\}, \\
\pi^{-1}(\underline{i}) \cap \operatorname{cosv}\left(\pi^{-1}(\underline{j})\right)=\left\{w_{0} \subsetneq \cdots \subsetneq w_{m} \mid a \in w_{0} \text { for some } a \leq i,\right. \\
\left.b \in w_{m} \text { for some } b \leq j\right\} .
\end{array}
$$

For an arbitrary

$$
d=\left(w_{0} \subsetneq \cdots \subsetneq w_{m}\right) \in \pi^{-1}(\underline{i}) \cap \operatorname{cosv}\left(\pi^{-1}(\underline{j})\right),
$$

the category $\pi^{-1}(j) / d$ has a terminal object: Just delete every $w_{r}$ that does not contain some $c \leq \bar{j}$. Thus, $\pi^{-1}(\underline{j}) \rightarrow \pi^{-1}(\underline{i}) \cap \operatorname{cosv}\left(\pi^{-1}(\underline{j})\right)$ is homotopy initial and the homotopy limits agree by Proposition 3.6.

Next we observe that $\pi^{-1}(\underline{i}) \cap \operatorname{cosv}\left(\pi^{-1}(\underline{j})\right) \subset \pi^{-1}(\underline{i})$ and $\pi^{-1}(\underline{i} \backslash \underline{j}) \rightarrow \pi^{-1}(\underline{i})$ are cosieves. Thus, the result follows by Proposition 3.7. 
Let $0 \leq k \leq n$ still be arbitrary. The following lemma will be proven in Section 6 .

Lemma 4.8 The nerves of the categories

- $\pi^{-1}(\mathcal{E})$ for every nonempty connected subcategory $\mathcal{E} \subseteq \underline{n}$ that is not $\underline{n} \backslash\{k\}$,

- $\pi^{-1}(i) \cap \operatorname{cosv}\left(\pi^{-1}(\underline{i-1})\right)$ for $i \geq 1$ if $k<n$ or $i<n-1$, and

- $\pi^{-1}((n-1) \rightarrow n) \cap \operatorname{cosv}\left(\pi^{-1}(\underline{n-2})\right)$ for $k=n \geq 2$

are (weakly) contractible.

The next lemma now follows easily.

\section{Lemma 4.9 The maps}

(i) $\operatorname{holim}_{\pi^{-1}(i)} F \rightarrow F(i)$,

(ii) $\operatorname{holim}_{\pi^{-1}(i)} F \rightarrow \operatorname{holim}_{\pi^{-1}(i) \cap \operatorname{cosv}\left(\pi^{-1}(\underline{i-1})\right)} F$ for $i \geq 1$ if $k<n$ or $i<n-1$,

(iii) $\operatorname{holim}_{\pi^{-1}((n-1) \rightarrow n)} F \rightarrow \operatorname{holim}_{\pi^{-1}((n-1) \rightarrow n) \cap \operatorname{cosv}\left(\pi^{-1}(n-2)\right)} F$ for $k=n$

are weak equivalences.

Proof As Nerve $\pi^{-1}(i) \simeq *$ by Lemma 4.8, Proposition 3.4 implies part (i). The same argument implies that source and target in (ii) are equivalent to $F(\{i\} \subset\{i-1, i\})$ and so (ii) follows from the 2-out-of-3 principle.

Recall that for $k=n$ the map $(n-1) \rightarrow n$ is a weak equivalence in $\underline{n}$. Thus, by the same argument, both source and target in (iii) are equivalent to $F(\{n-1\} \subset\{n-2, n-1\})$ and (iii) follows, again from the 2-out-of-3 principle.

We are now ready for the proof of Proposition 4.6.

Proof of Proposition 4.6 Assume first that $k<n$. By Lemma 4.9,

$$
\operatorname{holim}_{\pi^{-1}(i)} F \rightarrow \operatorname{holim}_{\pi^{-1}(i) \cap \operatorname{cosv}\left(\pi^{-1}(i-1)\right)} F
$$

is a weak equivalence for every $i \geq 1$. Thus, every homotopy pullback along this map is a weak equivalence, in particular, using Lemma 4.7, the map

$$
\operatorname{holim}_{\pi^{-1}(i)} F \rightarrow \operatorname{holim}_{\pi^{-1}\left({ }^{i-1}\right)} F .
$$

By Lemma 4.9, it follows that the composition of the maps

$$
\operatorname{holim}_{\pi^{-1}(\underline{n})} F \rightarrow \cdots \rightarrow \operatorname{holim}_{\pi^{-1}(\underline{0})} F \rightarrow F(0)
$$

is a weak equivalence as well. This shows Proposition 4.6 in the case $k<n$. 
The same arguments show that the map holim $\pi^{-1}(\underline{n-2)} F \rightarrow F(0)$ and the map from $\operatorname{holim}_{\pi^{-1}(n)} F \simeq \operatorname{holim}_{\pi^{-1}((n-1) \rightarrow n)} F \times_{\pi^{-1}((n-1) \rightarrow n) \cap \operatorname{cosv}\left(\pi^{-1}(n-2)\right)}^{h} \operatorname{holim}_{\pi^{-1}(n-2)} F$ to holim $_{\pi^{-1}(n-2)} F$ are weak equivalences. Therefore their composition

$$
\operatorname{holim}_{\pi^{-1}(\underline{n})} F \rightarrow F(0)
$$

is also a weak equivalence in this case.

As discussed above, Proposition 4.6 implies:

Theorem 4.10 For a fibration category $\mathcal{M}$, the simplicial space $N_{\xi} \mathcal{M}$ is Reedy fibrant.

We now want to deduce our main theorem from this. Recall to that purpose that a full subcategory $\mathcal{C} \subset \mathcal{M}$ is called homotopically full if $x \in \mathcal{C}$ and $x \simeq y$ in $\mathcal{M}$ already imply $y \in \mathcal{C}$. The crucial ingredient is the following theorem (known in this form at least to Zhen Lin Low).

Theorem 4.11 (Low, Mazel-Gee) Let $\mathcal{M}$ be a fibration category and $\mathcal{C} \subseteq \mathcal{M}$ be a homotopically full subcategory. Then a Reedy fibrant replacement of $N(\mathcal{C}$, we $\mathcal{C})$ is a complete Segal space.

Proof The full subcategory of fibrant objects $\mathcal{M}^{\circ}$ of $\mathcal{M}$ forms a category of fibrant objects in the sense of Brown. Set $\mathcal{C}^{\circ}=\mathcal{M}^{\circ} \cap \mathcal{C}$.

By [12, Theorem A.5], combined with [12, Lemma 3.7, Lemma 3.11 and Remark 2.9], $\mathcal{C}^{\circ}$ has a homotopical three-arrow calculus. By the main result of [13], this implies that a Reedy fibrant replacement of $N\left(\mathcal{C}^{\circ}, w^{\circ} \mathcal{C}^{\circ}\right)$ is a complete Segal space.

A theorem of Cisinski [12, Theorem A.3] implies that the inclusions

$$
\text { Nerve we }\left(\left(\mathcal{M}^{\circ}\right)^{\underline{p}}\right) \rightarrow \text { Nerve we }\left(\mathcal{M}^{\underline{p}}\right)
$$

are weak equivalences for all $p \geq 0$. As $\mathcal{C} \subseteq \mathcal{M}$ is homotopically full, this implies that the inclusions Nerve we $\left(\mathcal{C}^{\circ}\right) \underline{p} \rightarrow$ Nerve we $\mathcal{C} \underline{p}$ are also weak equivalences. Therefore, $N(\mathcal{C}$, we $\mathcal{C})$ and $N\left(\mathcal{C}^{\circ}\right.$, we $\left.\mathcal{C}^{\circ}\right)$ are Reedy equivalent. Thus, a Reedy fibrant replacement of $N(\mathcal{C}$, we $\mathcal{C})$ is a complete Segal space.

Remark 4.12 The theorem above was already proven for simplicial model categories in [18] and for partial model categories (hence all homotopically full subcategories of model categories) in [4]. 
Theorem 4.13 Every fibration category is fibrant in the Barwick-Kan model structure.

Proof Let $\mathcal{M}$ be a fibration category. The natural map $N \mathcal{M} \rightarrow N_{\xi} \mathcal{M}$ is a Reedy equivalence as shown in [3]. By Theorem 4.10, it follows that $N_{\xi} \mathcal{M}$ is a Reedy fibrant replacement of $N \mathcal{M}$ and therefore fibrant in the Rezk model structure by the last theorem. As fibrations in RelCat are defined via $N_{\xi}$, it follows that $\mathcal{M}$ is fibrant in RelCat.

A slight variant of the proof gives actually the following stronger theorem:

Theorem 4.14 Every homotopically full subcategory of a fibration category is fibrant in the Barwick-Kan model structure.

Proof Let $\mathcal{C}$ be a homotopically full subcategory of a fibration category $\mathcal{M}$. By Theorem 4.11, we only have to show that $N_{\xi} \mathcal{C}$ is Reedy fibrant.

For Theorem 4.10, we have checked the fibrancy criterion Proposition 4.5. More precisely, we have shown that $\mathcal{M}$ has the right lifting property with respect to all

$$
c \mathrm{Sd}^{2} \Lambda^{k}[n] \rightarrow \mathcal{K}\left(c \mathrm{Sd}^{2} \Lambda^{k}[n]\right),
$$

where the relative structure on $\mathcal{K}\left(c \mathrm{Sd}^{2} \Lambda^{k}[n]\right)$ is induced by an arbitrary relative structure on $\underline{n}$ such that $(n-1) \rightarrow n$ is a weak equivalence if $k=n$.

We now want to check this fibrancy criterion for $\mathcal{C}$. Choose a relative structure on $\underline{n}$ as above. Let $F: c \operatorname{Sd}^{2} \Lambda^{k}[n] \rightarrow \mathcal{C}$ be a functor and $G: \mathcal{K}\left(c \operatorname{Sd}^{2} \Lambda^{k}[n]\right) \rightarrow \mathcal{M}$ be an extension. Let $x \in c \operatorname{Sd}^{2} \Lambda^{k}[n]$ be arbitrary. As $G(x, 1) \simeq G(x, 0)=F(x)$ and $G\left(k_{c \mathrm{Sd}^{2} \Lambda^{k}[n]}\right) \simeq G(\{0\}, 1)$, the functor $G$ actually factors over $\mathcal{C}$.

We now want to indicate what happens if one considers cofibration categories instead of fibration categories. Define $\bar{\xi}=\xi_{i} \xi_{t}$. Then there is a functor

$$
N_{\bar{\xi}}: \text { RelCat } \rightarrow \text { ssSet, }
$$

where $N_{\bar{\xi}}(\mathcal{C})=\operatorname{RelCat}(\bar{\xi}(\check{\underline{p}} \times \underline{\hat{q}}), \mathcal{C})$ for a relative category $\mathcal{C}$. Barwick and Kan define in [3] a conjugate model structure on RelCat, where a morphism $f$ is a fibration or weak equivalence if and only if $N_{\bar{\xi}}(f)$ is in the Rezk model structure.

For our purposes, a cofibration category consists of a relative category $(\mathcal{M}$, we $\mathcal{M})$ together with a subcategory $\operatorname{cof} \mathcal{M} \subseteq \mathcal{M}$ such that $\left(\mathcal{M}^{\mathrm{op}},(\text { we } \mathcal{M})^{\mathrm{op}},(\operatorname{cof} \mathcal{M})^{\mathrm{op}}\right)$ is a fibration category. By [3, Theorem 6.4] a relative category $\mathcal{M}$ is fibrant in the conjugate model structure if and only if $\mathcal{M}^{\mathrm{op}}$ is fibrant in the usual Barwick-Kan model structure. We obtain:

Corollary 4.15 Every cofibration category is fibrant in the conjugate Barwick-Kan model structure. 


\section{General fibrancy criteria}

In this section, we will give criteria for the Reedy fibrancy of $N_{\xi} \mathcal{M}$, where $\mathcal{M}$ will be throughout an arbitrary relative category. This will culminate in Proposition 5.3, which is the relevant criterion for Section 4.

To use the notion of Reedy fibrancy, we have to view simplicial spaces now no longer as bisimplicial sets, but as simplicial objects in simplicial sets instead; more precisely, we view a bisimplicial set $K_{\bullet \bullet}$ now as a simplicial object $K_{\bullet}$ with $K_{m}=K_{m}$.

There are two ways to view a simplicial set as a simplicial space, a horizontal and a vertical one. For a simplicial set $K$, let $K^{h}$ be the simplicial space with $\left(K^{h}\right)_{m}=K$ for all $m$. Furthermore, let $K^{v}$ be the simplicial space with $\left(K^{v}\right)_{m}=K_{m}$, where we view a set as a discrete simplicial set. Note that $\Delta[n]^{h} \times \Delta[m]^{v} \cong \Delta[m, n]$; in particular,

$$
\operatorname{Hom}_{\mathrm{ssSet}}\left(\Delta[n]^{h} \times \Delta[m]^{v}, X_{\bullet \bullet}\right)=X_{m n} .
$$

Lemma 5.1 The simplicial space $N_{\xi} \mathcal{M}$ is Reedy fibrant if and only if $\mathcal{M}$ has the right lifting property in RelCat with respect to

$$
c \operatorname{Sd}^{2}\left(\Lambda^{k}[n] \times \Delta[m] \cup_{\Lambda^{k}[n] \times \partial \Delta[m]} \Delta[n] \times \partial \Delta[m]\right) \rightarrow c \operatorname{Sd}^{2}(\Delta[n] \times \Delta[m]),
$$

for all $n \geq 1, m \geq 0$ and $0 \leq k \leq n$, where the target inherits its relative structure by the identification with $\xi(\underline{\hat{n}} \times \underline{\underline{m}})$ and the relative structure on the source is induced by that on the target.

Proof The simplicial space $N_{\xi} \mathcal{M}$ is Reedy fibrant if and only if a lift exists in all diagrams

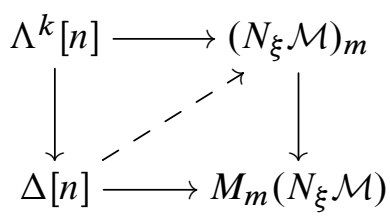

for all $n \geq 1, m \geq 0$ and $0 \leq k \leq n$. Here, $M_{m}$ denotes the matching object.

We have

$$
\left(N_{\xi} \mathcal{M}\right)_{m}=\operatorname{Map}\left(\Delta[m]^{v}, N_{\xi} \mathcal{M}\right)
$$

and

$$
M_{m} N_{\xi} \mathcal{M}=\operatorname{Map}\left(\partial \Delta[m]^{v}, N_{\xi} \mathcal{M}\right)
$$

with the map between them induced by the inclusion of the source; see [10, Chapter IV.3, pages 218-219]. In general, for simplicial spaces $X$ and $Y$, we take as mapping space $\operatorname{Map}(X, Y)$ the simplicial set with $l$-simplices $\operatorname{Hom}\left(X \times \Delta[l]^{h}, Y\right)$. 
By adjunction, a lift in the diagram above is now equivalent to a lift in the diagram

$$
\begin{aligned}
& \Lambda^{k}[n]^{h} \times \Delta[m]^{v} \cup_{\Lambda^{k}[n]^{h} \times \partial \Delta[m]^{v}} \Delta[n]^{h} \times \partial \Delta[m]^{v} \underset{\longrightarrow}{\longrightarrow} N_{\xi} \mathcal{M} \\
& \Delta[n]^{h} \times \Delta[m]^{v}
\end{aligned}
$$

and by another adjunction equivalent to a lift in the diagram:

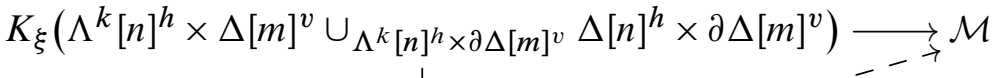

$$
\begin{aligned}
& K_{\xi}\left(\Delta[n]^{h} \times \Delta[m]^{v}\right)
\end{aligned}
$$

As explained in Section 2, this lifting problem is isomorphic to

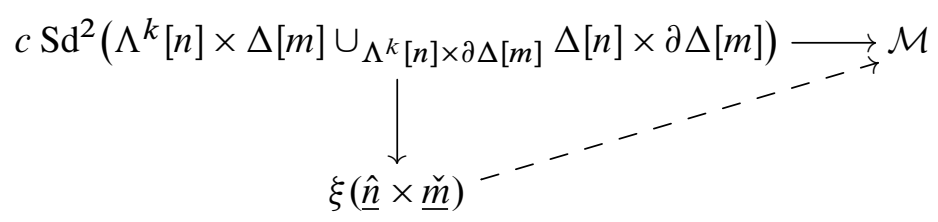

where the upper left corner inherits a relative structure from $\xi(\underline{\hat{n}} \times \underline{\underline{m}})$.

Lemma 5.2 The simplicial space $N_{\xi} \mathcal{M}$ is Reedy fibrant if $\mathcal{M}$ has the right lifting property with respect to all

$$
c \operatorname{Sd}^{2} \Lambda^{k}[n] \rightarrow \xi \underline{n},
$$

with $0 \leq k \leq n$ and $n \geq 1$, where $\underline{n}$ carries an arbitrary relative structure satisfying the following conditions:

- $\underline{n}$ has at least one nonidentity weak equivalence,

- $0 \rightarrow 1$ in $\underline{n}$ is a weak equivalence if $k=0$,

- $(n-1) \rightarrow n$ in $\underline{n}$ is a weak equivalence if $k=n$.

Here, $c \operatorname{Sd}^{2} \Lambda^{k}[n]$ inherits the relative structure from $\xi \underline{n}$.

Proof Denote by $I$ the collection of inclusions $c \operatorname{Sd}^{2} \Lambda^{k}[n] \rightarrow \xi \underline{n}$ of relative categories as described in the statement of the lemma. We want to show that

$c \operatorname{Sd}^{2}\left(\Lambda^{k}[n] \times \Delta[m] \cup_{\Lambda^{k}[n] \times \partial \Delta[m]} \Delta[n] \times \partial \Delta[m]\right) \rightarrow c \operatorname{Sd}^{2}(\Delta[n] \times \Delta[m])=\xi(\underline{\hat{n}} \times \underline{\underline{m}})$ 
is in $I$-cell, which means in our case that it can be built by iterative pushouts along maps in $I$. Then Lemma 5.1 implies that $\mathcal{M}$ having the right lifting property with respect to $I$ is sufficient for the Reedy fibrancy of $N_{\xi} \mathcal{M}$.

Recall that a marked simplicial set is a simplicial set $S$ with a subset $E \subset S_{1}$ of marked edges, containing all degenerate ones. Call an inclusion of marked simplicial sets with underlying map $\Lambda^{k}[n] \rightarrow \Delta[n]$

- an inner horn if $0<k<n$,

- a special left horn if $k=0$ and $0 \rightarrow 1$ is marked,

- a special right horn if $k=n$ and $(n-1) \rightarrow n$ is marked.

We denote by $J=J_{l}$ the collection of inner and special left horns and by $J_{r}$ the collection of inner and special right horns. We call a marked simplicial set maximally marked if all edges are marked and minimally marked if only the degenerate edges are marked.

Nerve and $c$ extend to an adjunction between relative categories and marked simplicial sets, compatible with forgetful functors. We define $\mathrm{Sd}^{2}$ on marked simplicial sets to be the unique colimit-preserving endofunctor such that $\operatorname{Sd}^{2}$ Nerve $\mathcal{C}=$ Nerve $\xi \mathcal{C}$ for $\mathcal{C}$ a relative poset.

As $c$ and $\mathrm{Sd}^{2}$ are left adjoints and therefore preserve pushouts, it is enough to show that the inclusion

$$
\phi=\phi_{k, n, m}: \Lambda^{k}[n] \times \Delta[m] \cup_{\Lambda^{k}[n] \times \partial \Delta[m]} \Delta[n] \times \partial \Delta[m] \rightarrow \Delta[n] \times \Delta[m]
$$

with $\Delta[n]$ maximally and $\Delta[m]$ minimally marked, is in $J$-cell for $k<n$ and in $J_{r}$-cell for $k>0$. Here, an edge is marked in the product if it is the product of two marked edges.

We will use the idea of the box product lemma of Dugger and Spivak [9, Appendix A]. Their proof essentially gives that for $k>0$ the map $\phi$ is in $J_{r}$-cell. Therefore, we will only show that for $k<n$ the map $\phi$ is in $J$-cell. Our proof will be dual to that of [9] and we will follow the approach there closely.

Let $Y=\Delta[n] \times \Delta[m]$ and let $Y^{0}=\Lambda^{k}[n] \times \Delta[m] \cup_{\Lambda^{k}[n] \times \partial \Delta[m]} \Delta[n] \times \partial \Delta[m]$. We will produce a filtration

$$
Y^{0} \subset Y^{1} \subset \cdots \subset Y^{m+1}=Y
$$

and prove that each $Y^{i} \rightarrow Y^{i+1}$ is in $J$-cell. 
Let us establish some notation. An $r$-simplex $y$ in $Y$ is determined by its vertices, and we can denote it in the form

$$
\left[\begin{array}{llll}
a_{0} & a_{1} & \cdots & a_{r} \\
u_{0} & u_{1} & \cdots & u_{r}
\end{array}\right]
$$

where $0 \leq a_{i} \leq a_{i+1} \leq n$ and $0 \leq u_{i} \leq u_{i+1} \leq m$, for $0 \leq i<r$. Indeed, an $r$-simplex in $\Delta[n] \times \Delta[m]$ corresponds to a functor $\underline{r} \rightarrow \underline{n} \times \underline{m}$. Faces and degeneracies are obtained by omitting or repeating columns. The simplex $y$ is thus degenerate if and only if two successive columns are identical. We will also call the $i$ th face "the $\left[\begin{array}{l}a_{i} \\ u_{i}\end{array}\right]$-face". Furthermore, we call the set $\left\{u_{0}, \ldots, u_{r}\right\}$ "the set of $u_{i}$ of $y$ ".

One checks that the simplex $y$ is an element of $Y^{0}$ if and only if it satisfies one of the following two conditions:

(i) $\left\{a_{0}, a_{1}, \ldots, a_{r}\right\}$ equals neither $\{0,1, \ldots, n\}$ nor $\{0,1, \ldots, n\} \backslash\{k\}$, or

(ii) $\left\{u_{0}, u_{1} \ldots, u_{r}\right\} \neq\{0,1, \ldots, m\}$.

Let $Y^{1}$ be the simplicial set generated (by faces and degeneracies) by the union of $Y^{0}$ together with all simplices that contain the vertex $\left[\begin{array}{c}k \\ m\end{array}\right]$, and in general let $Y^{i}$ be the simplicial set generated by the union of $Y^{i-1}$ together with all simplices containing $\left[\begin{array}{c}k \\ m-i+1\end{array}\right]$. Note that $Y^{m+1}=Y$ : Every simplex either contains some $\left[\begin{array}{c}k \\ m-i+1\end{array}\right]$ or is a face of such a simplex.

Our goal is to show that each inclusion $Y^{i} \rightarrow Y^{i+1}$ is in $J$-cell, and we will do this by producing another filtration

$$
Y^{i}=Y^{i}[n-1] \subset Y^{i}[n] \subset \cdots \subset Y^{i}[n+r]=Y^{i+1} .
$$

Notice that every simplex of $Y$ of dimension $n-1$ or less that contains $\left[\begin{array}{c}k \\ m-i\end{array}\right]$ actually lies in $Y^{0}$ as it satisfies condition (i). For $t>n-1$ we define $Y^{i}[t]$ to be the union of $Y^{i}[t-1]$ and all the faces of the nondegenerate simplices of $Y$ that have dimension $t$ and contain $\left[\begin{array}{c}k \\ m-i\end{array}\right]$ (and degeneracies of them). We claim that $Y^{i}[t] \rightarrow Y^{i}[t+1]$ is a cobase change of special left horn inclusions; justifying this will conclude our proof.

Let $y$ be a nondegenerate simplex of $Y$ of dimension $t+1 \geq n$ such that $y \in Y^{i}[t+1]$, but $y \notin Y^{i}[t]$; in particular, $y$ contains the vertex $\left[\begin{array}{c}k \\ m-i\end{array}\right]$. Then every face of $y$ except possibly for the $\left[\begin{array}{c}k \\ m-i\end{array}\right]$-face (ie that face obtained by leaving out this vertex) is contained in $Y^{i}[t]$. We must show that $Y^{i}[t]$ cannot contain this final face of $y$, and also that this final face is not the face of another nondegenerate simplex in $Y^{i}[t+1]$. Given the former, the latter is clear since two different simplices cannot have the same $\left[\begin{array}{c}k \\ m-i\end{array}\right]$-face. 
Now we want to show that the $\left[\begin{array}{c}k \\ m-i\end{array}\right]$-face $d y$ of $y$ is not in $Y^{i}[t]$. Write $y$ as

$$
\left[\begin{array}{llll}
a_{0} & a_{1} & \cdots & a_{t+1} \\
u_{0} & u_{1} & \cdots & u_{t+1}
\end{array}\right] .
$$

The column $\left[\begin{array}{c}k \\ m-i\end{array}\right]$ cannot be the last one in $y$ since $\left\{a_{0}, a_{1}, \ldots, a_{t+1}\right\}=\{0,1, \ldots, n\}$ and $k<n$. Consider the column in $y$ after $\left[\begin{array}{c}k \\ m-i\end{array}\right]$. The difference between this column and $\left[\begin{array}{c}k \\ m-i\end{array}\right]$ can neither in first nor in second entry exceed one as else $y \in Y^{0}$. The column cannot equal $\left[\begin{array}{c}k \\ m-i+1\end{array}\right]$ or $\left[\begin{array}{c}k \\ m-i\end{array}\right]$ since then $y \in Y^{i}$ or $y$ is degenerate. Thus it has to equal $\left[\begin{array}{c}k+1 \\ ?\end{array}\right]$. The second entry cannot be $m-i+1$ since then we could insert between these two columns an entry $\left[\begin{array}{c}k \\ m-i+1\end{array}\right]$ so that $y$ would be a face of a simplex in $Y^{i}$ and thus would itself be in $Y^{i}$. Therefore, this column has to be $\left[\begin{array}{c}k+1 \\ m-i\end{array}\right]$.

The set of $u_{i}$ in $d y$ is equal to the set of $u_{i}$ in $y$, namely $\left\{u_{1}, \ldots, u_{t+1}\right\}=\{1, \ldots, m\}$. Thus, $d y \notin Y^{0}$ as $y \notin Y^{0}$. Thus, $d y$ can only be in $Y^{i}[t]$ if it either contains $\left[\begin{array}{c}k \\ m-j\end{array}\right]$ for some $j \leq i$ or it is the $\left[\begin{array}{c}k \\ m-j\end{array}\right]$-face of another simplex with $j \leq i-1$. Either is absurd.

As $\left[\begin{array}{c}k \\ m-i\end{array}\right]$ was not the last column, we have proved that the inclusion $Y^{i}[t] \rightarrow Y^{i}[t+1]$ is a cobase change along inner and left horns, one horn inclusion for each $y$. If $\left[\begin{array}{c}k \\ m-i\end{array}\right]$ was actually the zeroth column (in the case in which we are filling a 0-horn), the edge from the zeroth vertex to the first vertex is marked as the second entries of both agree and the edge is therefore a product of a marked and a degenerate edge. Thus, $\phi$ is in $J$-cell.

For the next proposition, please recall the notations $\mathcal{K}(\mathcal{D})$ and $k_{\mathcal{D}}$ from the beginning of Section 4. Recall also from the discussion before Proposition 4.5 how a relative structure on $\underline{n}$ induces a relative structure on $\mathcal{K}\left(c \mathrm{Sd}^{2} \Lambda^{k}[n]\right)$ and hence by restriction also on $c \mathrm{Sd}^{\overline{2}} \Lambda^{k}[n]$.

Proposition 5.3 The simplicial space $N_{\xi} \mathcal{M}$ is Reedy fibrant if $\mathcal{M}$ has the right lifting property with respect to all

$$
c \mathrm{Sd}^{2} \Lambda^{k}[n] \rightarrow \mathcal{K}\left(c \mathrm{Sd}^{2} \Lambda^{k}[n]\right)
$$

with $0 \leq k \leq n$ and $n \geq 1$, where the relative structure on $c \operatorname{Sd}^{2} \Lambda^{k}[n]$ and the relative structure on $\mathcal{K}\left(c \mathrm{Sd}^{2} \Lambda^{k}[n]\right)$ are induced by an arbitrary relative structure on $\underline{n}$ satisfying the following conditions:

- $\underline{n}$ has at least one nonidentity weak equivalence,

- $0 \rightarrow 1$ in $\underline{n}$ is a weak equivalence if $k=0$,

- $n-1 \rightarrow n$ in $\underline{n}$ is a weak equivalence if $k=n$. 
Proof Fix $0 \leq k \leq n$ and equip $\underline{n}$ with a relative structure with at least one weak equivalence such that the map $0 \rightarrow 1$ in $\underline{n}$ is a weak equivalence if $k=0$ and $(n-1) \rightarrow n$ is a weak equivalence if $k=n$. Assume that $\mathcal{M}$ has the right lifting property with respect to

$$
c \operatorname{Sd}^{2} \Lambda^{k}[n] \rightarrow \mathcal{K}\left(c \operatorname{Sd}^{2} \Lambda^{k}[n]\right) .
$$

By Lemma 5.2, we only need to show that $\mathcal{M}$ also has the right lifting property with respect to

$$
c \mathrm{Sd}^{2} \Lambda^{k}[n] \rightarrow \xi \underline{n}
$$

We will proceed as in [15, Lemma 4.2], but we have to take extra care here since not all morphisms are weak equivalences.

Recall that $\mathcal{K}\left(c \mathrm{Sd}^{2} \Lambda^{k}[n]\right)$ is isomorphic to a full subposet $\mathcal{P}_{k}$ of $c \mathrm{Sd}^{2} \Delta[n]$, described as follows: The subposet $c \operatorname{Sd}^{2} \Lambda^{k}[n]$ of $c \operatorname{Sd}^{2} \Delta[n]$ consists of all those sequences $v_{0} \subsetneq \cdots \subsetneq v_{m}$ for which $v_{m} \neq \underline{n}$ and $v_{m} \neq \underline{n} \backslash\{k\}$. The subposet $\mathcal{P}_{k}$ of $c \operatorname{Sd}^{2} \Delta[n]$ consists of all sequences $v_{0} \subsetneq \cdots \subsetneq v_{m}$ in $c \operatorname{Sd}^{2} \Lambda^{i}[n]$, for each such sequence also the sequence $v_{0} \subsetneq \cdots \subsetneq v_{m} \subsetneq \underline{n}$, and finally the sequence consisting only of $\underline{n}$ (corresponding to $\left.k_{c \mathrm{Sd}^{2} \Lambda^{k}[n]} \in \mathcal{K}\left(c \mathrm{Sd}^{2} \Lambda^{k}[n]\right)\right)$.

It suffices to show that each relative functor defined on $\mathcal{P}_{k}$ can be extended to $c \operatorname{Sd}^{2} \Delta[n]$. We will give a retraction for the inclusion of $\mathcal{P}_{k}$ into $c \mathrm{Sd}^{2} \Delta[n]$, ie an order-preserving map $c \mathrm{Sd}^{2} \Delta[n] \rightarrow \mathcal{P}_{k}$, which is the identity on $\mathcal{P}_{k}$ and respects weak equivalences. This will complete the proof.

Observe that the only objects of $c \mathrm{Sd}^{2} \Delta[n]$ which are not in $\mathcal{P}_{k}$ are given by sequences in which $\underline{n} \backslash\{k\}$ occurs; more precisely, these are the sequences $\underline{n} \backslash\{k\}, \underline{n} \backslash\{k\} \subsetneq \underline{n}$, $w_{0} \subsetneq \cdots \subsetneq w_{l} \subsetneq \underline{n} \backslash\{k\}$ and $w_{0} \subsetneq \cdots \subsetneq w_{l} \subsetneq \underline{n} \backslash\{k\} \subsetneq \underline{n}$, where in the last two cases, $w_{0} \subsetneq \cdots \subsetneq w_{l}$ is a sequence of nonempty subsets of $\underline{n} \backslash\{k\}$.

The map $r: c \mathrm{Sd}^{2} \Delta[n] \rightarrow \mathcal{P}_{k}$ is described by

$$
A \mapsto\left\{\begin{array}{cl}
A & \text { if } A \in \mathcal{P}_{k}, \\
\underline{n} & \text { if } A=\underline{n} \backslash\{k\} \text { or } A=\underline{n} \backslash\{k\} \subsetneq \underline{n}, \\
w_{0} \subsetneq w_{1} \subsetneq \cdots \subsetneq w_{l} \subsetneq \underline{n} & \text { if } A=\left(w_{0} \subsetneq w_{1} \subsetneq \cdots \subsetneq w_{l} \subsetneq \underline{n} \backslash\{k\}\right) \text { or } \\
A & =\left(w_{0} \subsetneq w_{1} \subsetneq \cdots \subsetneq w_{l} \subsetneq \underline{n} \backslash\{k\} \subsetneq \underline{n}\right) .
\end{array}\right.
$$

Note that the assignment above covers all cases. Furthermore, the map takes only values in $\mathcal{P}_{k}$ and is by definition the identity on $\mathcal{P}_{k}$, and it is checked in [15, Lemma 4.2] that it is order-preserving. We have only to show that it preserves weak equivalences. As described in Section 2, weak equivalences are detected by the smallest element of the first set in the chain. This can only change by application of $r$ if $k=0$ and then it can 
change at most from 0 to 1 . As the morphism $0 \rightarrow 1$ is a weak equivalence if $k=0$, the retraction $r$ preserves weak equivalences.

This completes the proof of the proposition.

\section{Contractible subsets of simplices}

The aim of this section is to prove Lemma 4.8. Throughout this section, we use the notation of Section 4. This means that $n \geq 1$ is a fixed natural number and $0 \leq k \leq n$. Furthermore, $\mathcal{D}=c \operatorname{Sd}^{2} \Lambda^{k}[n]$ and $\pi: \mathcal{D} \rightarrow \underline{n}$ is the functor described in the discussion after Proposition 4.5. Moreover, $\operatorname{cosv}(\mathcal{C}) \subset \mathcal{D}$ denotes for a subcategory $\mathcal{C} \subset \mathcal{D}$ again the cosieve generated by $\mathcal{C}$, ie the full subcategory of all $d \in \mathcal{D}$ such that a morphism $c \rightarrow d$ in $\mathcal{D}$ with $c \in \mathcal{C}$ exists.

We will split up the statement of Lemma 4.8 into several lemmas.

Lemma 6.1 Let $\mathcal{E} \subset \underline{n}$ be a subcategory equal to neither $\underline{n} \backslash\{k\}$ nor $(\underline{n} \backslash\{k\}) \sqcup\{k\}$. Then the nerve of the category $\pi^{-1}(\mathcal{E})$ is weakly equivalent to the nerve of $\mathcal{E}$. In particular, if $\mathcal{E}$ is in addition nonempty and connected, the nerve of $\pi^{-1}(\mathcal{E})$ is contractible.

Proof We can assume that $\mathcal{E}$ is nonempty and connected. We have to show that Nerve $\pi^{-1}(\mathcal{E})$ is contractible. This is clear for $\mathcal{E}=\underline{n}$, so that we can assume $\mathcal{E} \neq \underline{n}$. Throughout this proof, we mean by $W_{\bullet}$ a nonempty chain $W_{0} \subsetneq \cdots \subsetneq W_{l}$ of nonempty subsets of $\underline{n}$ such that $W_{l}$ is neither $\underline{n}$ nor $\underline{n} \backslash\{k\}$.

Let $\mathcal{C} \subset \pi^{-1}(\mathcal{E})$ be the full subcategory of all those $W_{\bullet}$ with $W_{0} \subset \mathcal{E}$. We want to apply Quillen's Theorem A to show that the inclusion $\mathcal{C} \rightarrow \pi^{-1}(\mathcal{E})$ induces a weak homotopy equivalence on nerves. We have to show that for every $W_{\bullet} \in \pi^{-1}(\mathcal{E})$, the nerve of the undercategory $W_{\bullet} / \mathcal{C}$ is contractible. As $\pi^{-1}(\mathcal{E})$ is a poset, we can identify this undercategory with a subcategory of $\mathcal{C}$. With this identification, there is an adjunction

$$
\mathcal{D} \underset{\rho}{\stackrel{\lambda}{\rightleftarrows}} W_{\bullet} / \mathcal{C}
$$

where $\mathcal{D}$ is the poset of nonempty chains of nonempty subsets of $W_{0} \cap \mathcal{E}$, and $\lambda$ and $\rho$ are defined as follows: We define $\lambda\left(V_{\bullet}\right)$ as the concatenation $V_{\bullet} \subset W_{\bullet}$ and we define $\rho\left(V_{\bullet}\right)$ as $V_{0} \subsetneq \cdots \subsetneq V_{q}$, where $q \geq 0$ is the largest index such that $V_{q} \subset W_{0} \cap \mathcal{E}$; as $V_{\bullet} \in \mathcal{C}$, such an index must exist. By the adjunction, $\operatorname{Nerve} \mathcal{D} \simeq \operatorname{Nerve}\left(W_{\bullet} / \mathcal{C}\right)$. The nerve of $\mathcal{D}$ is the double-subdivision of a (nonempty) simplex and thus contractible. Thus, $\mathcal{C} \rightarrow \pi^{-1}(\mathcal{E})$ induces a weak equivalence on nerves. 
Let $\mathcal{D}^{\prime}$ be the full subcategory of $\pi^{-1}(\mathcal{E})$ of all chains $W_{\bullet}$ with $W_{l} \subset \mathcal{E}$. There is an obvious inclusion $\mathcal{D}^{\prime} \rightarrow \mathcal{C}$. This inclusion has a right adjoint $s: \mathcal{C} \rightarrow \mathcal{D}^{\prime}$ with $s\left(W_{\bullet}\right)=W_{0} \subsetneq \cdots \subsetneq W_{q}$ for $q \geq 0$ the largest index with $W_{q} \subset \mathcal{E}$. Thus,

$$
\text { Nerve } \pi^{-1}(\mathcal{E}) \simeq \text { Nerve } \mathcal{C} \simeq \text { Nerve } \mathcal{D}^{\prime},
$$

and we only need to show that Nerve $\mathcal{D}^{\prime}$ is contractible. As $\mathcal{E}$ is neither $\underline{n}$ nor $\underline{n} \backslash\{k\}$, we have Nerve $\mathcal{D}^{\prime} \cong \mathrm{Sd}^{2}$ Nerve $\mathcal{E} \simeq *$. This completes the proof.

Denote $\pi^{-1}(i) \cap \operatorname{cosv}\left(\pi^{-1}(\underline{i-1)})\right)$ for $i \geq 1$ for short by $X_{i}^{k}$. We want to show that the nerve of $X_{i}^{k}$ is contractible unless $k=n$ and $i$ equals $n-1$ or $n$.

Define $\bar{X}_{i}^{k}$ as the poset of chains $W_{0} \subsetneq \cdots \subsetneq W_{l}$ in $X_{i}^{k}$ such that $W_{0}=\{i\}$. There is a left adjoint $\lambda$ to the inclusion $\bar{X}_{i}^{k} \rightarrow X_{i}^{k}$, defined by

$$
\lambda\left(W_{0} \subsetneq \cdots \subsetneq W_{l}\right)=\left(\{i\} \subsetneq W_{0} \subsetneq \cdots \subsetneq W_{l}\right)
$$

for chains not in $\bar{X}_{i}^{k}$ and $\left.\lambda\right|_{\bar{X}_{i}^{k}}=\mathrm{id}_{\bar{X}_{i}^{k}}$. Thus, the nerves of $X_{i}^{k}$ and $\bar{X}_{i}^{k}$ are homotopy equivalent.

Lemma 6.2 We have

$$
\bar{X}_{i}^{k} \cong \begin{cases}c\left(\mathrm{Sd}^{2} \partial \Delta[n-1]\right) \backslash c\left(\mathrm{Sd}^{2} d_{01 \ldots(i-1)} \Delta[n-1]\right) & \text { for } k=i, \\ c\left(\mathrm{Sd}^{2} \Lambda^{k}[n-1]\right) \backslash c\left(\mathrm{Sd}^{2} d_{01 \ldots(i-1)} \Delta[n-1]\right) & \text { for } k<i, \\ c\left(\mathrm{Sd}^{2} \Lambda^{k-1}[n-1]\right) \backslash c\left(\mathrm{Sd}^{2} d_{01 \ldots(i-1)} \Delta[n-1]\right) & \text { for } k>i\end{cases}
$$

Here, $d_{01 \ldots(i-1)}$ is the face operator induced by the map

$$
[n-1-i] \rightarrow[n-1], \quad m \mapsto m+i ;
$$

the face is understood to be empty if $i=n$. Furthermore, if $\mathcal{E} \subset \mathcal{C}$ is a full subcategory, we denote by $\mathcal{C} \backslash \mathcal{E}$ the full subcategory of $\mathcal{C}$ with objects $\mathrm{Ob} \mathcal{C} \backslash \mathrm{Ob} \mathcal{E}$.

Proof By deleting all occurrences of $i$, we can identify $\bar{X}_{i}^{k}$ with the poset of all chains $W_{\bullet}=\left(W_{0} \subsetneq \cdots \subsetneq W_{l}\right)$ in $\underline{n}$ such that $W_{l}$ is neither $\underline{n} \backslash\{i\}$ nor $\underline{n} \backslash\{i, k\}$, $W_{l}$ contains a $j$ satisfying $j<i$, and none of the sets contains $i$.

Denote by $Y_{i}^{k}$ the poset of all $W_{\bullet}$ such that $W_{l}$ is neither $\underline{n} \backslash\{i\}$ nor $\underline{n} \backslash\{i, k\}$ and none of the sets contains $i$. We have

$$
Y_{i}^{k} \cong \begin{cases}c \operatorname{Sd}^{2} \partial \Delta[n-1] & \text { for } k=i \\ c \operatorname{Sd}^{2} \Lambda^{k}[n-1] & \text { for } k<i \\ c \operatorname{Sd}^{2} \Lambda^{k-1}[n-1] & \text { for } k>i\end{cases}
$$


The poset of all chains not containing a $j$ satisfying $j<i$ forms the subcategory $c \mathrm{Sd}^{2} d_{01 \ldots(i-1)} \Delta[n-1]$.

Lemma 6.3 Let $L$ be a (topologically realized) simplicial complex and $K \subset L$ be a full subcomplex. This means that any collection $v_{0}, \ldots, v_{i}$ of 0 -simplices in $K$ spans a simplex in $K$ if it spans a simplex in $L$. Then

$$
L \backslash K \simeq L \backslash \operatorname{st}(K),
$$

where $\operatorname{st}(K)$ is the open star of $K$, the union of all interiors of simplices in $L$ having nonempty intersection with $K$.

Proof It is enough to find a deformation retraction of $L \backslash K$ onto $L \backslash \operatorname{st}(K)$. First consider the case $L=\Delta[i]$ and $K=d_{j+1 \ldots i} \Delta[i]=\Delta[j] .{ }^{4}$ In this case, we use

$$
(\Delta[i] \backslash \Delta[j]) \times I \rightarrow \Delta[i] \backslash \Delta[j], \quad\left(t_{0}: \cdots: t_{i}, s\right) \mapsto\left(s t_{0}: \cdots: s t_{j}: t_{j+1}: \cdots: t_{i}\right) .
$$

These are homogeneous coordinates, ie we implicitly normalize. For a general subsimplex $K$ of $\Delta[i]$, we multiply exactly the homogeneous coordinates corresponding to the possibly nonzero coordinates in $K$ by $s$ instead.

In the general case, it is enough to define the map $(L \backslash K) \times I \rightarrow L \backslash K$ on every single (half-open) simplex in a way compatible with restriction to subsimplices. As $K$ is full, the intersection of $K$ with an arbitrary simplex $\Delta$ in $L$ is a subsimplex of $\Delta$. Thus, we can use the map described above.

These two lemmas imply the following result:

Lemma 6.4 The nerve of the category $\pi^{-1}(i) \cap \operatorname{cosv}\left(\pi^{-1}(\underline{i-1})\right)$ is contractible for $i \geq 1$ unless $k=n$ and $i$ equals $n-1$ or $n$.

Proof Observe first the following two simple facts:

(1) Nerve $c$ Nerve $\cong$ Nerve.

(2) If $\mathcal{E} \subset \mathcal{C}$ is a full subcategory such that $|\operatorname{Nerve} \mathcal{C}|$ is a simplicial complex, then $|\operatorname{Nerve}(\mathcal{C} \backslash \mathcal{E})| \cong|\operatorname{Nerve}(\mathcal{C})| \backslash \operatorname{st}(|\operatorname{Nerve}(\mathcal{E})|)$.

\footnotetext{
${ }^{4}$ We use here the same symbol for the topological simplex as for the simplex in simplicial sets, but this should not be confusing.
} 
From the last two lemmas, we then obtain

$$
\mid \text { Nerve } \bar{X}_{i}^{k} \mid \simeq \begin{cases}\partial \Delta[n-1] \backslash d_{01 \ldots(i-1)} \Delta[n-1] & \text { for } k=i \\ \Lambda^{k}[n-1] \backslash d_{01 \ldots(i-1)} \Delta[n-1] & \text { for } k<i \\ \Lambda^{k-1}[n-1] \backslash d_{01 \ldots(i-1)} \Delta[n-1] & \text { for } k>i\end{cases}
$$

Denote the right-hand side by $Z_{i}^{k}$.

In the case $k=i$, we remove a $\Delta[n-i-1]$ from $\partial \Delta[n-1] \cong S^{n-2}$. Thus, $Z_{i}^{k}$ is contractible unless $k=i=n$. Indeed, choose a vertex $j$ in $\Delta[n-i-1]$. Then there is a deformation retraction of $Z_{i}^{k}$ onto $d_{j} \Delta[n-1]$, with possibly a subsimplex removed; this is contractible.

In the case $k \neq i$, the simplex $d_{01 \ldots(i-1)} \Delta[n-1]$ equals neither the vertex $k$ for $k<i$ nor the vertex $k-1$ for $k>i$ (as $d_{01 \ldots(i-1)} \Delta[n-1]$ is just one point only if $\left.i=n-1\right)$. If the tip of the horn is not in $d_{01 \ldots(i-1)} \Delta[n-1]$, then a linear deformation towards the tip is the required contraction of $Z_{i}^{k}$. If the tip of the horn is in $d_{01 \ldots(i-1)} \Delta[n-1]$, deforming away from the tip gives a homotopy equivalence of $Z_{i}^{k}$ to $\partial \Delta[n-2]$ with one (nonempty) subsimplex removed. The same argument as before gives that this is contractible.

It remains to show the following lemma:

Lemma 6.5 If $k=n \geq 2$, then the nerve of $Y=\pi^{-1}((n-1) \rightarrow n) \cap \operatorname{cosv}\left(\pi^{-1}(\underline{n-2})\right)$ is contractible.

Proof Let $W_{\bullet}=\left(W_{0} \subsetneq \cdots \subsetneq W_{l}\right)$. Then $W_{\bullet} \in Y$ if and only if

- $W_{0}$ contains no elements but $(n-1)$ and $n$,

- $W_{l} \neq \underline{n}$ and $W_{l} \neq \underline{n-1}$,

- there exists $j \in W_{l}$ with $j<n-1$.

Denote the subcategory of those $W_{\bullet}$ in $Y$ with $W_{0}=\{n\}$ by $Y_{0}$. By deleting $n$, this can be identified with $c \operatorname{Sd}^{2} \partial \Delta[n-1] \backslash\{n-1\}$.

Denote the subcategory of those $W_{\bullet}$ in $Y$ with $W_{0}=\{n-1\}$ by $Y_{1}$. By deleting $(n-1)$, this can be identified with $c \operatorname{Sd}^{2} \Lambda^{n-1}[n-1] \backslash\{n-1\}$.

Denote the subcategory of those $W_{\bullet}$ in $Y$ with $W_{0}$ or $W_{1}$ equaling $\{n-1, n\}$ by $Y_{2}$. This is isomorphic to $\left(c \mathrm{Sd}^{2} \partial \Delta[n-2]\right) \times(c \mathrm{Sd} \Delta[1])$, where the second coordinate corresponds to $W_{0}$ being $\{n\},\{n-1, n\}$ or $\{n-1\}$. 
The intersection $Y_{0} \cap Y_{2}$ is given by all the $W_{\bullet}$ in $Y$ with $W_{0}=\{n\}$ and $W_{1}=\{n-1, n\}$. By deleting all entries of the form $(n-1)$ or $n$, the intersection $Y_{0} \cap Y_{2}$ is isomorphic to $c \mathrm{Sd}^{2} \partial \Delta[n-2]$. By a similar argument, $Y_{1} \cap Y_{2} \cong c \mathrm{Sd}^{2} \partial \Delta[n-2]$.

In total, we see that

$$
\text { Nerve } Y=\text { Nerve } Y_{0} \cup_{\mathrm{Sd}^{2} \partial \Delta[n-2]} \text { Nerve } Y_{2} \cup_{\mathrm{Sd}^{2} \partial \Delta[n-2]} \text { Nerve } Y_{1} \text {. }
$$

By the identifications above, this is after geometric realization homeomorphic to

$$
D^{n-2} \cup_{S^{n-3}} S^{n-3} \times I \cup_{S^{n-3}} S^{n-3} \times I .
$$

This colimit in turn is homeomorphic to $D^{n-2}$, which is contractible.

\section{References}

[1] C Barwick, On left and right model categories and left and right Bousfield localizations, Homology, Homotopy Appl. 12 (2010) 245-320 MR

[2] C Barwick, D M Kan, A characterization of simplicial localization functors and a discussion of DK equivalences, Indag. Math. 23 (2012) 69-79 MR

[3] C Barwick, D M Kan, Relative categories: another model for the homotopy theory of homotopy theories, Indag. Math. 23 (2012) 42-68 MR

[4] C Barwick, D Kan, From partial model categories to $\infty$-categories, preprint (2013) Available at http://math.mit.edu/ clarkbar/papers.html

[5] J E Bergner, Complete Segal spaces arising from simplicial categories, Trans. Amer. Math. Soc. 361 (2009) 525-546 MR

[6] W Chachólski, J Scherer, Homotopy theory of diagrams, Mem. Amer. Math. Soc. 736, Amer. Math. Soc., Providence, RI (2002) MR

[7] D-C Cisinski, Catégories dérivables, Bull. Soc. Math. France 138 (2010) 317-393 MR

[8] D-C Cisinski, Invariance de la $K$-théorie par équivalences dérivées, J. K-Theory 6 (2010) 505-546 MR

[9] D Dugger, D I Spivak, Mapping spaces in quasi-categories, Algebr. Geom. Topol. 11 (2011) 263-325 MR

[10] P G Goerss, J F Jardine, Simplicial homotopy theory, Progress in Mathematics 174, Birkhäuser, Basel (1999) MR

[11] A Joyal, M Tierney, Quasi-categories vs Segal spaces, from "Categories in algebra, geometry and mathematical physics" (A Davydov, M Batanin, M Johnson, S Lack, A Neeman, editors), Contemp. Math. 431, Amer. Math. Soc. (2007) 277-326 MR

[12] Z L Low, Cocycles in categories of fibrant objects, preprint (2015) arXiv 
[13] Z L Low, A Mazel-Gee, From fractions to complete Segal spaces, Homology Homotopy Appl. 17 (2015) 321-338 MR

[14] J Lurie, Higher topos theory, Annals of Mathematics Studies 170, Princeton University Press (2009) MR

[15] L Meier, V Ozornova, Fibrancy of partial model categories, Homology Homotopy Appl. 17 (2015) 53-80 MR

[16] D G Quillen, Homotopical algebra, Lecture Notes in Mathematics 43, Springer (1967) MR

[17] A Radulescu-Banu, Cofibrations in homotopy theory (2006) arXiv

[18] C Rezk, A model for the homotopy theory of homotopy theory, Trans. Amer. Math. Soc. 353 (2001) 973-1007 MR

[19] C Schommer-Pries, Does the classification diagram localize a category with weak equivalences?, MathOverflow (2012) Available at http://tinyurl.com/q-93139

[20] M A Shulman, Set theory for category theory, preprint (2008) arXiv

[21] K Szumiło, Two models for the homotopy theory of cocomplete homotopy theories, preprint (2014) arXiv

[22] R W Thomason, Cat as a closed model category, Cahiers Topologie Géom. Différentielle 21 (1980) 305-324 MR

[23] B Toën, M Vaquié, Moduli of objects in dg-categories, Ann. Sci. École Norm. Sup. 40 (2007) 387-444 MR

Mathematisches Institut, University of Bonn

Endenicher Allee 60, D-53115 Bonn, Germany

lmeier@math.uni-bonn.de

http://www.math.uni-bonn.de/people/lmeier

Received: 20 April 2015 Revised: 1 April 2016 